\title{
Disaccharidase activity in the small intestine of gerbils (Meriones unguiculatus) during primary and challenge infections with Giardia lamblia
}

\author{
M BELOSEVIC, G M FAUBERT, AND J D MACLEAN
}

From the Department of Zoology, The University of Alberta CW-312 Biological Sciences Building, Edmonton, Alberta, Canada, Institute of Parasitology of McGill University, Ste. Anne de Bellevue, Quebec, Canada, and Tropical Disease Centre, Montreal General Hospital, Montreal, Quebec, Canada.

SUMmaRY The sequence of changes in the activity of six disaccharidases in the small intestine of gerbils during primary and secondary $G$ lamblia infections was examined. The primary $G$ lamblia infection induced a transient reduction in disaccharidase activity which was related to the highest trophozoite burden in the small intestine. During the primary exposure, a $30 \%$ to $85 \%$ decrease in the activity of enzymes was observed on days 10 and 20 after infection. Secondary exposure of gerbils to $G$ lamblia caused a sharp decrease in disaccharidase activity as early as $24 \mathrm{~h}$ after challenge. The reduction in the enzyme activity was not influenced by the size of the challenge inoculum and occurred even when there were no live trophozoites in the small intestine. Disaccharidase deficiency could also be induced by challenge with the soluble extract of the trophozoites. Multiple challenge administrations of $G$ lamblia trophozoites to gerbils induced a persistent disaccharidase deficiency. The results indicate that disaccharidase deficiency associated with the primary $G$ lamblia infection probably represents a direct effect of the parasite on the brush border of the small intestine. On the other hand, the observed disaccharidase deficiency in the secondary $G$ lamblia infection appears to be induced by the local immune responses of the host.

Various abnormalities of small bowel pathology have been identified consistently in human giardiasis and these include disaccharidase deficiencies, ${ }^{12}$ increased intraepithelial lymphocyte counts ${ }^{34}$ and, in patients with malabsorption crypt hyperplasia with short villi and increased lamina propria cellularity. ${ }^{5}$ Low serum calcium and folate concentrations were consistent features in elderly people having giardiasis and aroused suspicion of underlying malabsorption. ${ }^{6} \mathrm{~A}$ decrease in the disaccharidase activity has been observed in vitro. Mouse mucosal cells incubated with $G$ lamblia trophozoites showed a reduction in the activities of lactase, sucrase, and maltase. ${ }^{?}$

We have recently described an animal model for giardiasis using gerbils and $G$ lamblia isolated from humans. ${ }^{8}$ In this study, we examined the progression

Address all correspondence: Dr M Belosevic. Department of Zoology, CW-312 Biosciences Building, University of Alberta, Edmonton, Alberta, Canada T6G 2E9.

Accepted for publication 14 February 1989. of changes in the activity of six disaccharidases in the small intestine of gerbils during primary and challenge infections with G lamblia.

\section{Methods}

PARASITES

Trophozoites of $G$ lamblia, WB isolate, originally obtained from a symptomatic patient ${ }^{4}$ (American type culture collection no 30957) were used for experimental infections. Trophozoites were grown axenically in filter sterilised Diamond's TYI-S-33 medium using the procedures described previously. ${ }^{\prime \prime}$

ANIMALS

Six to 10 week old male Mongolian gerbils (Meriones unguiculatus) were obtained from Tumblebrook Farms Ltd. (West Brookfield, Ma). The procedures for the maintenance of Giardia free animal colony have been described previously. ${ }^{811}$ 
INOCULATIONG I.AMBLIA TROPHOZOITES TO GERBILS

Two hundred thousand trophozoites of $G$ lamblia were administered to gerbils either orally ${ }^{x}$ or by the duodenal injection technique of Belosevic and Dick. ${ }^{12}$

QUANTIFICATION OF TROPHOZOITES IN THE

SMALL INTESTINE

The number of trophozoites per gerbil and their location in the small intestine were determined by the technique of Belosevic et al..$^{13}$

PREPARATION OF THE SOLUBLE EXTRACT OF TROPHOZOITES

Trophozoites (48-96 h culture) were sedimented by centrifugation at $200 \mathrm{~g}$ for five minutes and washed three times in PBS at $200 \mathrm{~g}$ for $10 \mathrm{~min}$. Trophozoites were disrupted by sonication in PBS. After sonication, the suspension was dialysed using Spectrapor membrane (Spectrum Medical Industries Inc, Los Angeles, California) against PBS for $24 \mathrm{~h}$ at $4^{\circ} \mathrm{C}$. Protein concentration of each extract was determined using Bio-Rad protein determination kit (BioRad Chemical Co, Richmond, Ca). This extraction method yielded approximately $0.5 \mathrm{mg}$ of $\mathrm{G}$ lamblia protein from $2 \times 10^{7}$ trophozoites.

MEASUREMENT OF THE VILLUS: CRYPT RATIO The method of Roberts-Thomson et $a l^{14}$ for the determination of villus to crypt ratio in murine giardiasis was used except for the following modification. Small intestine was removed, opened longitudinally, fixed in $10 \%$ formalin, and processed in paraffin for light microscopy. All histologic specimens (haematoxylin-eosin $8 \mu \mathrm{m}$ ) were examined at $400 \times$ and measurements made with an ocular micrometer. The height of the villi and crypts was determined on 10 representative specimens of infected and uninfected gerbils.

MEASUREMENT OF INTESTINAL

DISACCHARIDASES

Preparation of intestinal homogenate

Gerbils were killed by cervical dislocation and the small intestine was removed and divided into four equal sections. The segments were placed in ice cold distilled water and flushed with $50 \mathrm{ml}$ distilled water using a syringe fitted with a cannula. The intestinal segments were then placed on top of an ice cold ground glass slab and were slit longitudinally. The mucosa was scraped from the intestine with a glass microscope slide, weighed (wet weight), and placed in 4 volumes of ice cold distilled water. The mucosal scrapings were homogenised using glass/Teflon homogeniser ( 10 strokes, using a Con Torque power unit at maximum speed; Eberbach Corp, Ann Arbor, $\mathrm{Mi}$. The homogenates were then centrifuged at 400 $g$ for five minutes in order to remove larger cell debris. Cell debris obtained during the preparation of homogenates from uninfected, infected and challenged gerbils was assayed and found to have marginal disaccharidase activity which was not significantly different between the experimental groups. After centrifugation, the homogenates were stored in liquid nitrogen in $0.2 \mathrm{ml}$ aliquots until used in the assays.

\section{Reagents}

All substrates were obtained commercially. Glucose (D-16) and sucrose (S-5B) were purchased from Fisher Scientific Co. (Montreal, Que). Maltose (M-5885), cellobiose (C-7252), lactose (L-3750), trehalose (T-5251) and isomaltose (I-7253) were obtained from Sigma Chemical Co (St Louis, Mo). Chemicals for the glucose oxidase reagent were also obtained commercially. Sigma 7-9 Tris buffer (T-1378), Triton X-100 (X-100), o-dianisidine (D-9143), peroxidase (P-8000) and glucose oxidase (G-1262) were obtained from Sigma.

\section{Assay for intestinal disaccharidases}

Disaccharidase activity of mucosal scrapings was assessed using modified glucose oxidase peroxidase enzyme system. ${ }^{15}$ The assay was done in 96-well Linbro microtitre plates (Flow Laboratories, Toronto, Ont). The assay consisted of adding to each well $0.01 \mathrm{ml}$ appropriately diluted mucosal sample and $0.01 \mathrm{ml}$ substrate buffer solution of a disaccharidase to be measured in triplicate. The plates were incubated at $37^{\circ} \mathrm{C}$ in humidified atmosphere for 60 $\mathrm{min}$. After incubation, $0 \cdot 3 \mathrm{ml}$ of tris-glucose oxidase (TGO) reagent was added to each well and the plates were incubated for an additional $60 \mathrm{~min}$. The plates were read at $414 \mathrm{~nm}$ using a microplate reader (Titertec Multiscan, Flow). For each assay, eight reagent blanks $(0.02 \mathrm{ml}$ distilled water and $0.3 \mathrm{ml}$ TGO reagent) and two glucose standard series $(2,6$ and $10 \mu \mathrm{g}$ glucose) were also done. Throughout this paper, disaccharidase activity will be expressed as units/g of the mucosal sample.

STATISTICAL ANALYSIS

Data were analysed by Student's $t$ test and one-way analysis of variance (ANOVA), using BIOM statistical programs for the IBM personal computer. The probability level of $\mathrm{p}<0.05$ was considered significant.

\section{Results}

COURSE OF INFECTION

Trophozoite colonisation of the small intestine of 
gerbils inoculatd orally and by duodenal injection were compared. The number of trophozoites that colonised the small intestine during the infection was similar in orally and duodenally inoculated gerbils. Highest trophozoite loads in the gut were observed on days 10 and 15 after infection in both groups. On day 15 after infection, the mean number of trophozoites in orally inoculated gerbils was $4.8(1.9) \times 10^{\circ} /$ gerbil and that of duodenally inoculated gerbils was $6 \cdot 6(1 \cdot 5) \times 10^{6} /$ gerbil. The number of trophozoites in the small intestine sharply decreased after day 20 of infection in both orally and duodenally inoculated animals.

Mean body weight of gerbils in experimental and control groups at the beginning of the experiments was 42 (3) g and 41 (2) g, respectively. At the end of the experiments the mean body weight of gerbils infected with $G$ lamblia was 63 (5) $\mathrm{g}$ and that of control uninfected animals was 67 (4) g. There were no differences in mean body weight between infected and uninfected gerbils ( $p>0.05$, one-way analysis of variance).

\section{MEASUREMENT OF VILLUS/CRYPT RATIO}

\section{DURING PRIMARY INFECTION}

The villus to crypt ratio in the duodenum of gerbils infected with $2 \times 10^{5}$ giardia trophozoites was determined every five days for a period of 35 days (Fig. 1). A significant decrease in the villus to crypt ratio occurred at day 10 to 20 after infection ( $p<0 \cdot 05$, oneway analysis of variance). At 25 to 35 days, however, the villus to crypt ratio returned to normal, a period which corresponds to the elimination of the trophozoites from the duodenum.

\section{MEASUREMENT OF INTESTINAL}

\section{DISACCHARIDASES}

Disaccharidase activity during the primary infection A $30 \%$ to $85 \%$ decrease in the activity of all disaccharidases, except cellobiase, was observed during the acute phase of the infection (Fig. 2). The decrease in the activity of the enzymes was related to the period of highest trophozoite burden in the small intestine. Significantly lower activities of maltase, isomaltase and sucrase were detected on days 10 and 20 of the primary infection. The activity of these disaccharidases returned to normal levels during the elimination phase of the infection (days 30 and 40). The decrease in trehalase activity occurred later (day 20 after infection), and persisted longer (up to day 30 after infection), when compared with that of maltase, isomaltase and sucrase.

The decrease in disaccharidase activity was observed in all sections of the small intestine (data not shown). Isomaltase activity in the small intestine of gerbils was consistently higher than either maltase

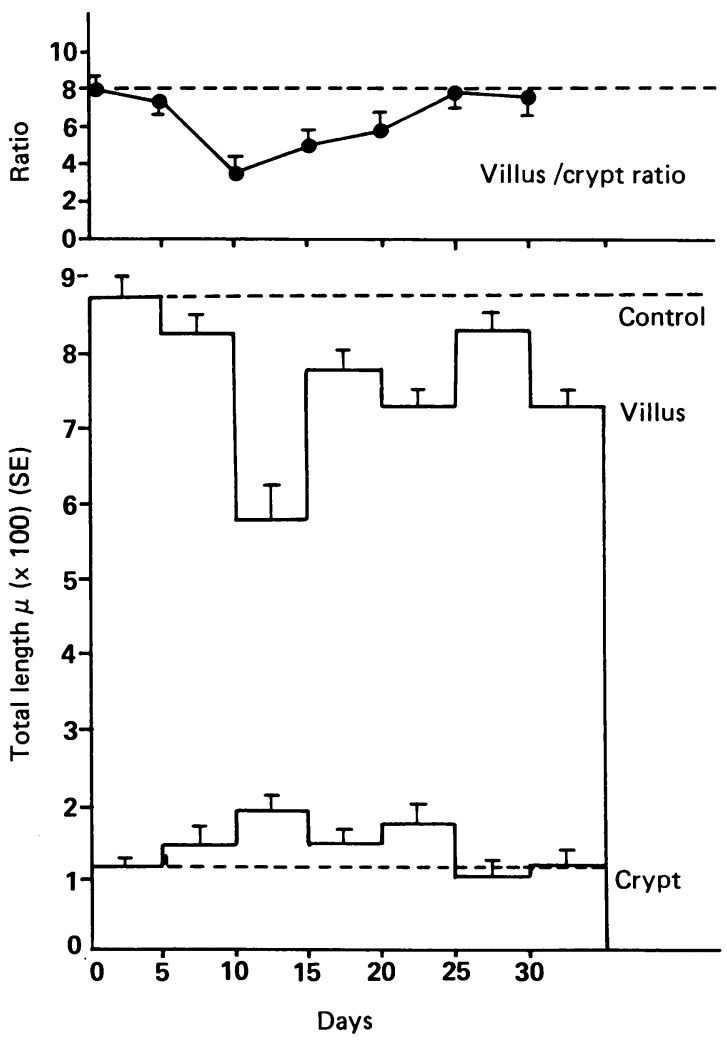

Fig. 1 Villus to crypt ratio of gerbil duodenum infected with $2 \times 10^{5}$ Giardia lamblia trophozoites. Mean values of 10 infected and 10 controls are given.

or sucrase activity, which is the opposite to that observed in man. The difference in the enzyme activity profiles may be due to the animal species used. Thus, $G$ lamblia infection induced transient reduction in maltase, isomaltase, sucrase and trehalase evident during the highest parsite density in the small intestine. With a low number of trophozoites present in the intestine (before day 10) or with a decrease in the number of organisms on days 30 and 40 , the activities of these disaccharidases returned to normal levels. On the other hand, lactase activity was significantly reduced during the acute and elimination phases of the infection and was not related to the trophozoite burden in the small intestine (Fig. 2).

\section{Disaccharidase activity at various times after a challenge infection}

Challenge inoculation of gerbils with $G$ lamblia trophozoites resulted in a sharp decrease in disaccharidase activity in the small intestine (Fig. 3). This reduction in enzyme activity occurred in the absence of trophozoites as the organisms were not observed in 

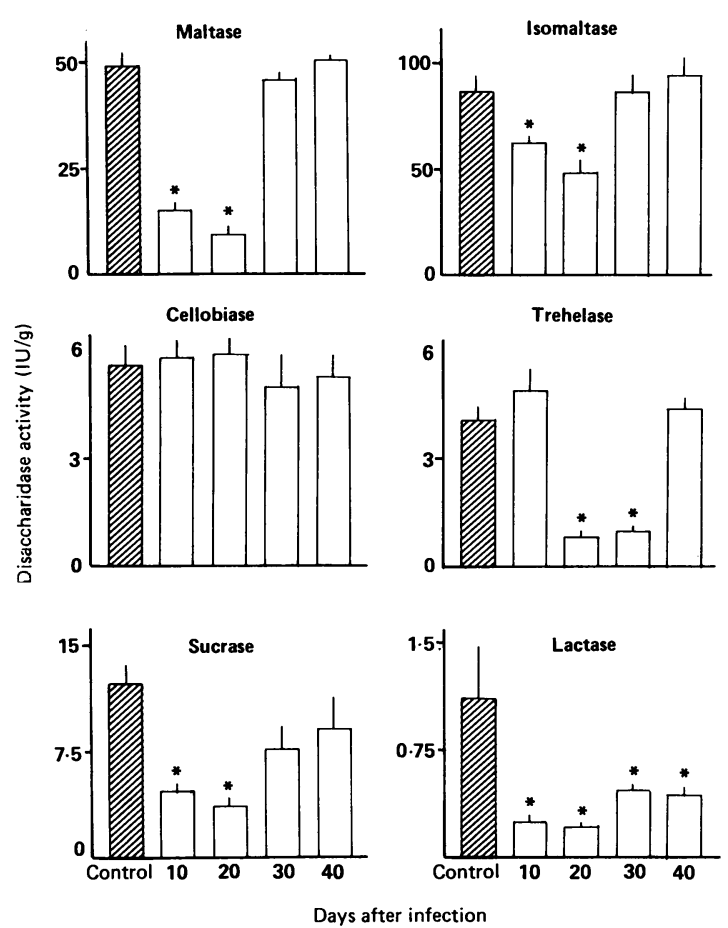

Fig. 2 Disaccharidase activity during the primary infection in the small intestine of gerbils infected with Giardia lamblia trophozoites. Activity of the enzymes was measured in $\mathrm{U} / \mathrm{g}$ tissue for the eight infected and eight non-infected control gerbils per test day. Infection dose was $2 \times 10^{\text {s }}$ trophozoites/ gerbil. Control values for different test periods were not significantly different (one-way ANOVA), hence the data were combined for analysis ( $n=40$ gerbils). Hatched bars: disaccharidase activity in the small intestine of age matched uninfected gerbils. $\left(^{*}\right)$ significantly different $(p<0 \cdot 05$, oneway ANOVA) when compared with the non-infected group.

the gut of all challenged gerbils. Sucrase and trehalase activity profiles after a challenge were similar (Fig. 3). For example, a significant reduction in sucrase and trehalase activity was observed as early as day 1 after challenge and on days 2, 4 and 8 after challenge infection. The activities of sucrase and trehalase returned to normal levels by day 16 and 30 , however, after the challenge infection, indicating that this depression in disaccharidase activity was transient. Similar results were obtained for lactase activity except that significantly lower activity was also observed on day 16 after challenge (Fig. 3). The decrease in disaccharidase activity of similar magnitude was also observed in posterior small intestine.

A reduction in disaccharidase activity upon challenge was also observed long after the primary infection. When gerbils were challenged on day 150 after the primary infection, a significantly lower

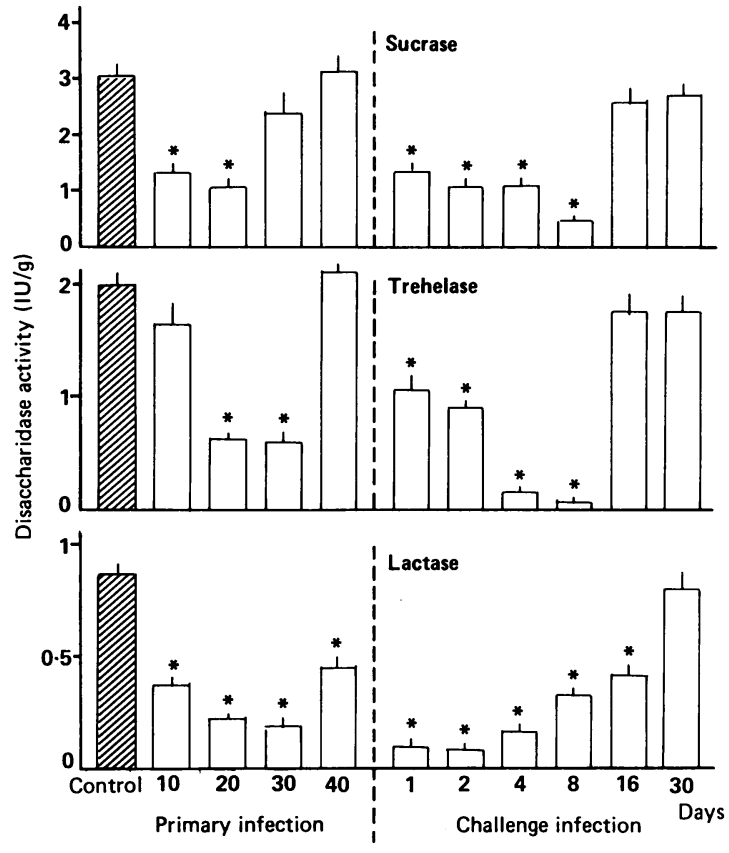

Fig. 3 Disaccharidase activity in the small intestine of gerbils during the primary infection and at various times after a challenge infection with trophozoites of Giardia lamblia. Each bar on the graph represents the mean disaccharidase activity (SEM) of six gerbils. Infection dose for the primary infection was $2 \times 10^{5}$ trophozoites/gerbil. Gerbils were challenged orally with $2 \times 10^{5}$ trophozoites each on day 40 after the primary infection. Hatched bars: disaccharidase activity in the small intestine of age-matched uninfected gerbils. $\left({ }^{*}\right)$ significantly different $(p<0 \cdot 05$, oneway ANOVA) when compared with the non-infected group.

sucrase, trehalase and lactase activity was observed on day 4 after the challenge when compared with agematched animals which did not receive a challenge infection.

Disaccharidase activity in the small intestine of gerbils given different challenge doses

The size of the challenge inoculum did not significantly affect the degree of disaccharidase deficiency (data not shown). Enzyme activity after challenge with different numbers of trophozoites was similar between gerbils given low doses $\left(5 \times 10^{4}, 1 \times 10^{5}\right)$ and those given high doses $\left(8 \times 10^{5}, 1 \cdot 6 \times 10^{\circ}\right)$. It should be noted, however, that except for cellobiase and lactase, the activity of the remainder of disaccharidases in challenged animals was significantly lower when compared with that in gerbils on day 40 after the primary infection. 
Table 1 Disaccharidase activity in the small intestine of gerbils after repeated challenge infections with Giardia lamblia trophozoites*

\begin{tabular}{|c|c|c|c|c|c|c|}
\hline $\begin{array}{l}\text { Number of } \\
\text { challenge infections }\end{array}$ & $\begin{array}{l}\text { Disacchar } \\
\text { Maltase }\end{array}$ & \multicolumn{5}{|c|}{ Disaccharidase activity (U/g) (mean (SEM) } \\
\hline No challenge & $57 \cdot 3(4 \cdot 9)$ & $71 \cdot 3(7 \cdot 3)$ & $5 \cdot 1(0 \cdot 4)$ & $12 \cdot 1(1 \cdot 1)$ & $3 \cdot 8(0 \cdot 3)$ & $0 \cdot 5(0 \cdot 1)$ \\
\hline 1 & $16 \cdot 8(2 \cdot 9)$ & $18 \cdot 8(3 \cdot 5)$ & $4 \cdot 8(0 \cdot 5)$ & $2 \cdot 6(0.9)$ & $0.7(0 \cdot 6)$ & $0.4(0 \cdot 1)$ \\
\hline 2 & $20 \cdot 8(2 \cdot 9)$ & $19 \cdot 8(2.9)$ & $4.4(0 \cdot 5)$ & $2 \cdot 2(0 \cdot 7)$ & $0 \cdot 6(0 \cdot 3)$ & $0 \cdot 3(0 \cdot 1)$ \\
\hline 3 & $19 \cdot 1(2 \cdot 4)$ & $21 \cdot 6(3 \cdot 1)$ & $5 \cdot 1(0 \cdot 7)$ & $1.9(0 \cdot 7)$ & $1 \cdot 0(0 \cdot 3)$ & $0 \cdot 5(0 \cdot 1)$ \\
\hline 4 & $21.5(1.8)$ & $18 \cdot 8(3 \cdot 3)$ & $4 \cdot 7(0 \cdot 6)$ & $2 \cdot 1(0 \cdot 5)$ & $0 \cdot 6(0 \cdot 1)$ & $0.4(0.2)$ \\
\hline
\end{tabular}

*For each group six gerbils were inoculated orally with $2 \times 10^{s}$ trophozoites each. On day 40 after the primary infection and on 10 day intervals thereafter, gerbils were challenged with $2 \times 10$ ' trophozoites each. For all groups, the disaccharidase activity was measured on day 4 after the last challenge infection. Except for cellobiase, all groups were significantly different $(\mathrm{p}<0 \cdot 05$, Student's $t$ test $)$ when compared with the no challenge group.

Disaccharidase activity in the small intestine of gerbils after repeated challenge infections

Repeated challenge inoculations of gerbils with $G$ lamblia trophozoites resulted in persistent disaccharidase deficiency. As shown in Table 1, the activities of maltase, isomaltase, sucrase, trehalase and lactase remained low after 2, 3, and 4 challenge inoculations given on 10 day intervals. The data indicate that frequent re-exposure of gerbils to $G$ lamblia results in continuous low enzyme activity long after the primary infection in the absence of trophozoites in the small intestine.

\section{Disaccharidase activity after challenge with the soluble extract of trophozoites}

As shown in Table 2, challenge of gerbils previously exposed to $G$ lamblia with the soluble extract of trophozoites resulted in significant reduction in disaccharidase activity. This decrease in enzyme activity was comparable to that induced by challenge with

Table 2 Disaccharidase activity after challenge with the soluble extract of trophozoites

\begin{tabular}{|c|c|c|c|c|}
\hline \multirow[t]{2}{*}{ Group* } & \multicolumn{4}{|c|}{ Disaccharidase activity (units/g) (mean (SEM) } \\
\hline & Maltase & Sucrase & Trehelase & Lactase \\
\hline No challenge & $54 \cdot 2(4 \cdot 3)$ & $14 \cdot 8(1 \cdot 3)$ & $4 \cdot 6(1 \cdot 7)$ & $0 \cdot 6(0 \cdot 1)$ \\
\hline Challenge with & & & & \\
\hline trophozoites & $13 \cdot 8(1 \cdot 5)$ & $3 \cdot 2(0 \cdot 9)$ & $0 \cdot 4(0 \cdot 5)$ & $0.4(0 \cdot 1)$ \\
\hline $\begin{array}{l}\text { Challenge with } \\
\text { extract }\end{array}$ & & & & \\
\hline $0.5 \mathrm{mg}$ & $18 \cdot 7(1 \cdot 6)$ & $1.9(0 \cdot 8)$ & $0 \cdot 3(0 \cdot 2)$ & $0.4(0 \cdot 2)$ \\
\hline $1.0 \mathrm{mg}$ & $16 \cdot 5(2 \cdot 0)$ & $2 \cdot 1(1 \cdot 0)$ & $0.4(0 \cdot 2)$ & $0 \cdot 4(0 \cdot 1)$ \\
\hline $2 \cdot 0 \mathrm{mg}$ & $17 \cdot 2(1 \cdot 6)$ & $3 \cdot 2(0 \cdot 9)$ & $0.4(0 \cdot 1)$ & $0 \cdot 3(0 \cdot 2)$ \\
\hline
\end{tabular}

*Five gerbils per group were infected with $2 \times 10$ ' trophozoites each on day 0 . On day 40 after the primary infection, gerbils were challenged with $2 \times 10$ trophozoites or were given orally $0.5 \mathrm{mg}$ of the soluble extract of trophozoites at a rate of $0.5 \mathrm{mg} / \mathrm{gerbil} / \mathrm{day}$. Four days after the last administration of the extract, disaccharidase activity was measured. All groups were significantly different ( $p<0 \cdot 05$, Student's $t$ test) when compared with the no challenge group. live trophozoites. The amount of the soluble extract administered did not affect the degree of disaccharidase deficiency, because the reduction in enzyme activity was similar between animals challenged with $0.5 \mathrm{mg}$ of the soluble extract and those challenged with $2 \mathrm{mg}$. The results show that the reduction in maltase, sucrase, trehalase and lactase activity upon challenge occurred in the absence of live $G$ lamblia trophozoites.

\section{Discussion}

There is enormous variability in both the clinical features and pathological changes associated with giardiasis in man. The degree of pathological damage to the small intestine has been correlated to the symptoms. ${ }^{+16}$ At present, we have little knowledge of the relative contributions of parasite and host related factors to the pathogenesis of giardiasis. For example, changes caused directly by the parasite, such as the damage to the microvillous border of the small intestine, ${ }^{17}$ may be related to the parasite burden in the gut. On the other hand, changes resulting from the host response can be identified by manipulating host factors such as immunity and nutrition. The gerbil $G$ lamblia model for giardiasis, ${ }^{8}$ provides the means whereby these components can be analysed separately.

In this study we have examined the progression of changes in the activity of six disaccharidases in the small intestine of gerbils during primary and secondary infections with G lamblia. Because the majority of trophozoites were located in the upper small intestine of gerbils during the infection, we measured enzyme activities in the duodenum, proximal and distal jejunum and ileum. The results show that $G$ lamblia induced a transient decrease in disaccharidase activity during the acute phase of the primary infection. This decrease in the enzyme activity was related temporally to the highest trophozoite burden in the small intestine, and was observed in all regions 
of the gut during the acute phase of the primary infection. There were also differences in the activity profiles of the enzymes. For example, the reduction in maltase, isomaltase, and sucrase activity was observed on days 10 and 20 after infection, whereas that of trehalase occurred later (days 20 and 30 of infection). A notable exception was lactase activity which remained low during both the acute and elimination phases of the infection and was not related to the trophozoite burden in the small intestine. Our results support, in part, those of Gillon et $a{ }^{18}{ }^{18}$ who showed that maltase, sucrase and lactase levels in the duodenum of mice infected with $G$ muris were reduced significantly during the second week after infection. These authors also reported that lactase activity in the duodenum returned to normal levels by week 10 after infection. This was not observed in our study, as lactase levels remained low during both the acute and elimination phases of the infection. The discrepancy in the findings may be due to different species of the parasite and different host used.

This is the first study that has examined disaccharidase activity after a secondary exposure to Giardia. Challenge inoculation of gerbils with $G$ lamblia trophozoites resulted in a sharp and immediate decrease in the activity of the enzymes in both anterior and posterior small intestine. Lower activities of sucrase and trehalase were observed until day 8 and lactase until day 16 after challenge. This reduction in the enzyme activity was not dependent on the size of the challenge inoculum and it occurred in the absence of trophozoites, as the organisms were not observed in the small intestine of any of the challenged gerbils. Moreover, challenge of gerbils previously exposed to $G$ lamblia with the soluble extract of trophozoites resulted in a significant reduction in disaccharidase activity, comparable with that induced by challenge with live trophozoites. These results indicate that a secondary exposure of gerbils to $G$ lamblia resulted in disaccharidase deficiency similar in magnitude to that observed during the primary $G$ lamblia infection.

The decrease in disaccharidase activity upon challenge in the apparent absence of trophozoites in the small intestine as well as after administration of the soluble extract of trophozoites suggest that disaccharidase deficiency may be controlled by both parasite and host related factors. During the primary $G$ lamblia infection, the disaccharidase deficiency associated with giardiasis is likely to represent a direct effect of the parasite on the brush border. In this case, maximal reduction in enzyme activity should correlate with the highest trophozoite load in the small intestine. Our results support this hypothesis because greatest reduction in disaccharidase levels occurred during the acute phase of the infection.

On the other hand, the reduction in enzyme activity upon challenge with live trophozoites or the soluble extract of the trophozoites suggests that disaccharidase deficiency may be caused by the local immune response of the host. It is known that gerbils previously exposed to $G$ lamblia are resistant to reinfection for at least eight months after the primary infection. ${ }^{\circ}$ We have recently shown that the resistance of gerbils to reinfection could occur in both the absence or presence of occult $G$ lamblia infection. ${ }^{1 "}$ These findings suggest that the immune response of the host is involved in the resistance to Giardia and that this resistance is of relatively long duration. It is interesting to note that secondary exposure of gerbils to $G$ lamblia as long as 150 days after the primary infection results in disaccharidase deficiency of similar magnitude to that observed during the primary infection. Our findings indicate that frequent re-exposure of the host to Giardia may affect the nutritional status of the host by reducing the carbohydrate uptake. It remains to be determined whether re-exposure to other gastrointestinal parasites would result in disaccharidase deficiency in host's intestinal mucosa.

This work was supported by the Medical Research Council of Canada (MRC no 8146) the National Sciences and Engineering Research Council of Canada (NSERC no 9374) and Alberta Heritage Foundation for Medical Research (AHFMR no 71090). Research at the Institute of Parasitology is supported by NSERC and FCAR.

\section{References}

1 Jennings W, Rowland R, Hecker R, Gibson GE, Fitch RJ, Reid DP. The significance of lower jejunal disaccharidase levels. Aust NZ J Med 1976; 6: 556-60.

2 Duncombe VM, Bolin TD, Davis AE, et al. Histopathology in giardiasis: a correlation with diarrhoea. Aust NZ J Med 1978; 8: 392-6.

3 Ferguson A, McClure JP, Townley RRW. Intraepithelial lymphocyte counts in small intestinal biopsies from children with diarrhoca. Acta Pediatr Scand 1976; 65: 541-6.

4 Wright SG, Tomkins AM. Quantification of the lymphocyte infiltrate in jejunal epithelium in giardiasis. Clin Exp Immunol 1977; 29: 408-12.

5 Yardley JH, Takano J, Hendrix TR. Epithelial and other mucosal lesions of the jejunum in giardiasis. Jejunal biopsy studies. Bull Johns Hopkins Hosp 1964; 115: $389-406$.

6 Beaumont DM, James OFW. Unsuspected giardiasis as a cause of malnutrition and diarrhoea in the elderly. Br Med J 1986; 293: 554.

7 Anand BS, Chaudhary A, Jyothi A, Yadev RS, Baveja 
UK. Experimental examination of the direct damaging effects of $G$ lamblia on intestinal mucosal scrapings of mice. Trans $R$ Soc Trop Med Hyg 1985; 79: 613-7.

8 Belosevic M, Faubert GM, MacLean JD, Law C, Croll NA. Giardia lamblia infections in mongolian gerbils: an animal model. J Infect Dis 1983; 147: 222-6.

9 Smith PD, Gillin FD, Spira WM, Nash TE. Chronic giardiasis: studies on drug sensitivity, toxin production and host immune response. Gastroenterology 1982; 83: 797-803.

10 Belosevic M, Faubert GM, Croll NA, MacLean JD. Giardia lamblia: axenic growth in autoclaved and filtered Diamond's TYI-S-3 medium. Can J Zool 1982; 60: 1673-5.

11 Belosevic M, Faubert GM, Skamene E, MacLean JD. Susceptibility and resistance of inbred mice to Giardia muris. Infect Immun 1984; 44: 282-6.

12 Belosevic M, Dick TA. Trichinella spiralis: comparison with an arctic isolate. Exp Parasitol 1980; 49: 266-76.

13 Belosevic M. Faubert GM. Giardia muris: correlation between oral dosage, course of infection and trophozoite distribution in the mouse small intestinc. Exp Parasitol 1983; 56 : 93.

14 Roberts-Thomson IC, Stevens DP, Mahmoud AAF, Warren KS. Giardiasis in the mouse: an animal model. Gastroenterology 1976; 71: 57-61.

15 Dahlqvist A. Assay for intestinal disaccharidases. Anal Biochem 1968; 22: 99-107.

16 Wright SG, Tomkins AM, Ridlcy DS. Giardiasis: clinical and therapeutic aspects. Gut 1977; 18: 343-50.

17 Erlandsen SL, Chase DG. Morphological alterations in the microvillous border of villous epithelial cells produced by intestinal microorganisms. Am J Clin Nutr 1974; $27: 1277$.

18 Gillon J, Thamery DA, Ferguson A. Features of small intestinal pathology (cpithelial cell kinctics, intraepithelial lymphocytes, disaccharidases) in a primary Giardia muris infection. Gut 1982; 23: 498-506.

19 Lewis PD Jr, Belosevic M, Faubert GM, Curthoys L, MacLean JD. Cortisone-induced recrudescence of $\mathrm{G}$ lamblia infections in gerbils. Am J Trop Med Hyg 1987; 36: $33-40$. 